\title{
Laparoscopic surgery for colorectal cancers complicated by Leriche syndrome: A report of three cases
}

\author{
KOJI MURONO, SOICHIRO ISHIHARA, KAZUSHIGE KAWAI, HIROSHI NAGATA, MANABU KANEKO, \\ KAZUHITO SASAKI, KOJI YASUDA, KENSUKE OTANI, TAKESHI NISHIKAWA, TOSHIAKI TANAKA, \\ TOMOMICHI KIYOMATSU, KEISUKE HATA, HIROAKI NOZAWA and TOSHIAKI WATANABE
}

Department of Surgical Oncology, Faculty of Medicine, The University of Tokyo, Tokyo 113-8655, Japan

Received November 30, 2016; Accepted June 6, 2017

DOI: 10.3892/ol.2017.6391

\begin{abstract}
Leriche syndrome is a disease of aortoiliac occlusion, which causes diminished femoral pulses, impotence and claudication. As blood flow to the rectum is also decreased in Leriche syndrome, reconstruction with anastomosis may be complicated by ischemia when performing rectal cancer surgery. The inferior epigastric arteries often provide collateral circulation to the lower limbs in patients with Leriche syndrome, therefore, attention should be paid not to injure them during trocar insertion when performing laparoscopic surgeries. The present study is a report on three cases of patients with colorectal cancer who were successfully treated with laparoscopic surgeries. The first case was of a 71-year-old man with rectal cancer. A preoperative computed tomography (CT) scan revealed occlusion of the aorta below the origin of the inferior mesenteric artery. The blood flow to the lower limbs was supplied through collateral arteries, including the inferior epigastric arteries and the deep circumflex iliac arteries. A laparoscopic Hartmann's operation was performed successfully following marking of the inferior epigastric arteries using ultrasonography to avoid damaging them during trocar insertion. The second case involved a 70-year-old man with three colorectal cancers of the transverse and sigmoid colon and rectum. A CT scan revealed occlusion of the aorta below the origin of the renal arteries. Laparoscopic assisted low anterior resection and left hemicolectomy with colostomy were performed. The final patient was a 61-year-old man with rectal cancer. As the right internal iliac artery was patent, the patient underwent laparoscopic assisted low anterior resection. All the patients were discharged from the hospital without complications. It is important to visualize the image the blood flow via CT angiography and to mark collateral arteries
\end{abstract}

Correspondence to: Dr Koji Murono, Department of Surgical Oncology, Faculty of Medicine, The University of Tokyo, 7-3-1 Hongo, Tokyo 113-8655, Japan

E-mail: muronok-sur@h.u-tokyo.ac.jp

Key words: colorectal cancer, Leriche syndrome, case report, computed tomography angiography, occlusion of aorta using ultrasonography preoperatively in patients with Leriche syndrome for whom laparoscopic surgery was planned for to treat colorectal cancer.

\section{Introduction}

Leriche syndrome is a disease of aortoiliac occlusion, which causes diminished femoral pulses, impotence, and claudication $(1,2)$. Hypertension, diabetes mellitus, hyperlipidemia, and smoking are risk factors for the disease (3). When performing colorectal cancer surgeries for patients with Leriche syndrome, the following considerations must be addressed. First, the blood flow to the rectum is also reduced because of occlusion of the internal iliac arteries and/or inferior mesenteric artery (IMA), and therefore, reconstruction with anastomosis may be complicated by ischemia when performing rectal cancer surgery. Second, the blood flow to the lower limb is supplied by collateral arteries, such as the inferior epigastric artery and deep circumflex iliac artery (4), and therefore, attention should be paid not to injure them during trocar insertion when performing laparoscopic surgeries.

Here, we report three rare cases of colorectal cancers complicated by Leriche syndrome, which were successfully treated with laparoscopic surgery. Informed consent was obtained from all patients and the publication of the case reports was approved by institutional ethics committee.

\section{Case report}

Case 1. A 71-year-old man underwent total colonoscopy to evaluate a chief complaint of melena and weight loss. Colonoscopy revealed rectal cancer (Fig. 1). Risk factors for arteriosclerosis for the patient included diabetes mellitus, hypertension, and smoking (30 times per day, from ages 20 to 54 years old). The ankle-brachial index (ABI) was 0.32 on the right side and 0.36 on the left side. A preoperative computed tomography (CT) scan revealed occlusion of the aorta just below the origin of the IMA (Fig. 2). The blood flow of the lower limbs was supplied through the inferior epigastric arteries and deep circumflex iliac arteries. While the IMA was patent, the internal iliac arteries were occluded bilaterally. A laparoscopic Hartmann's operation without anastomosis was performed because the IMA, which was the only blood supply 
of the rectum, would be sacrificed via lymph node dissection, and ischemia of the remnant rectum was anticipated. The inferior epigastric arteries had developed as collateral arteries. Therefore, these arteries were preoperatively marked using ultrasonography to avoid injury of the arteries during trocar insertion (Fig. 3). The ABI did not decrease (0.36 on the right side and 0.50 on the left side) and the patient was discharged from the hospital on postoperative day 14 without complications.

The CT scan performed 6 months after the surgery revealed that the enhancement of the remnant rectum was remained. However, the blood flow was supplied from the small collateral artery (Fig. 4).

Case 2. A 70-year-old man was admitted to our hospital because of bowel occlusion due to the cancer of the transverse colon. Colonoscopy revealed three colorectal cancers, located in the transverse colon, sigmoid colon, and rectum. The risk factor for arteriosclerosis for this patient was smoking (30 times per day, from ages 40 to 70 years old). ABI was 0.41 on the right side and 0.45 on the left side. A preoperative CT scan revealed occlusion of the aorta just below the origin of the renal arteries (Fig. 5). The blood flow of the lower limbs was supplied through the inferior epigastric arteries and deep circumflex iliac artery. Because the IMA and both internal iliac arteries were occluded, laparoscopic left hemicolectomy and rectal resection with colostomy were performed. The inferior epigastric arteries were important as collateral arteries, and were preoperatively marked using ultrasonography to avoid injury. The ABI did not change, and the patient was discharged from the hospital on postoperative day 18 , without complications.

Colonoscopy performed postoperatively revealed no ischemic changes or stenosis in the remnant rectum.

Case 3. A 61-year-old man underwent total colonoscopy to evaluate a chief complaint of melena, revealing rectal cancer. Risk factors for arteriosclerosis for this patient included diabetes mellitus, hypertension, hyperlipidemia, and smoking (40 times per day, from age 25 years to age 57 years). The ABI was 0.77 on the right side and 0.50 on the left side. A preoperative CT scan revealed occlusion of the left common iliac artery (Fig. 6). The blood flow to the left lower limb was supplied through the inferior epigastric artery and deep circumflex iliac artery. Because the right internal iliac artery was patent, he underwent laparoscopic low anterior resection with anastomosis. The left inferior epigastric artery was very important as a collateral artery. Therefore, the artery was preoperatively marked using ultrasonography to avoid injury. The patient had an uncomplicated postoperative course and was discharged from the hospital on postoperative day 18 .

Postoperative colonoscopy revealed no stenosis or ischemic change in anastomosis.

\section{Discussion}

The blood flow to the rectum is mainly supplied from the IMA and bilateral internal iliac arteries, and the main blood supply was interrupted in cases 1 and 2 . Impaired blood supply is considered one of the causes of anastomotic
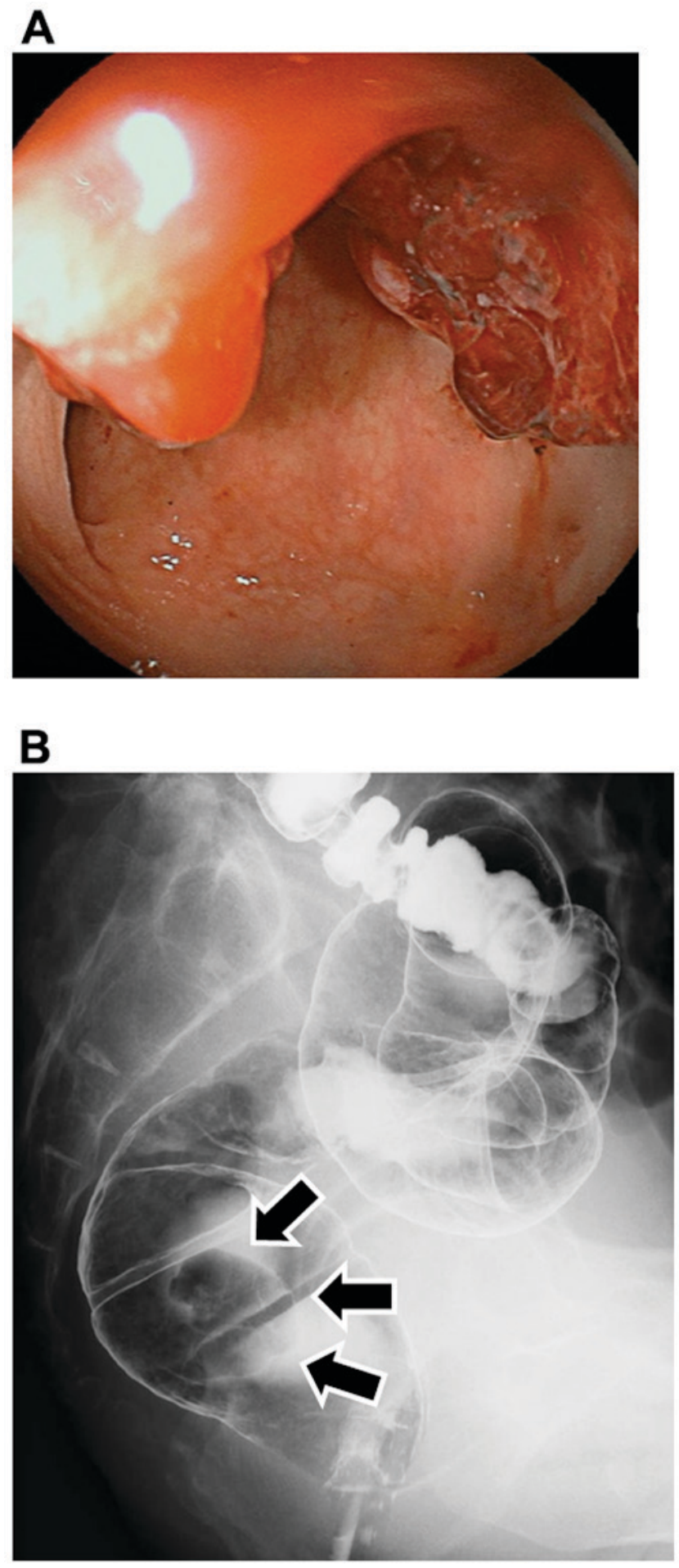

Figure 1. Case 1: A 71-year-old man with melena and weight loss (A) Colonoscopy revealed low rectal cancer. Biopsy revealed well differentiated adenocarcinoma. (B) Barium enema also revealed a low rectal tumor $3 \mathrm{~cm}$ in size (arrow).

leakage (5). Although some institutions use fluorescence imaging with indocyanine green to evaluate intestinal perfusion (6-8), it remains difficult to evaluate the risk of anastomotic leakage accurately. Therefore, Hartmann's operations were performed in two cases. Although impaired blood supply from the IMA and bilateral internal iliac arteries sometimes result in intestinal ischemia (9), no necrosis or stenosis of the remaining rectum was observed, 
A

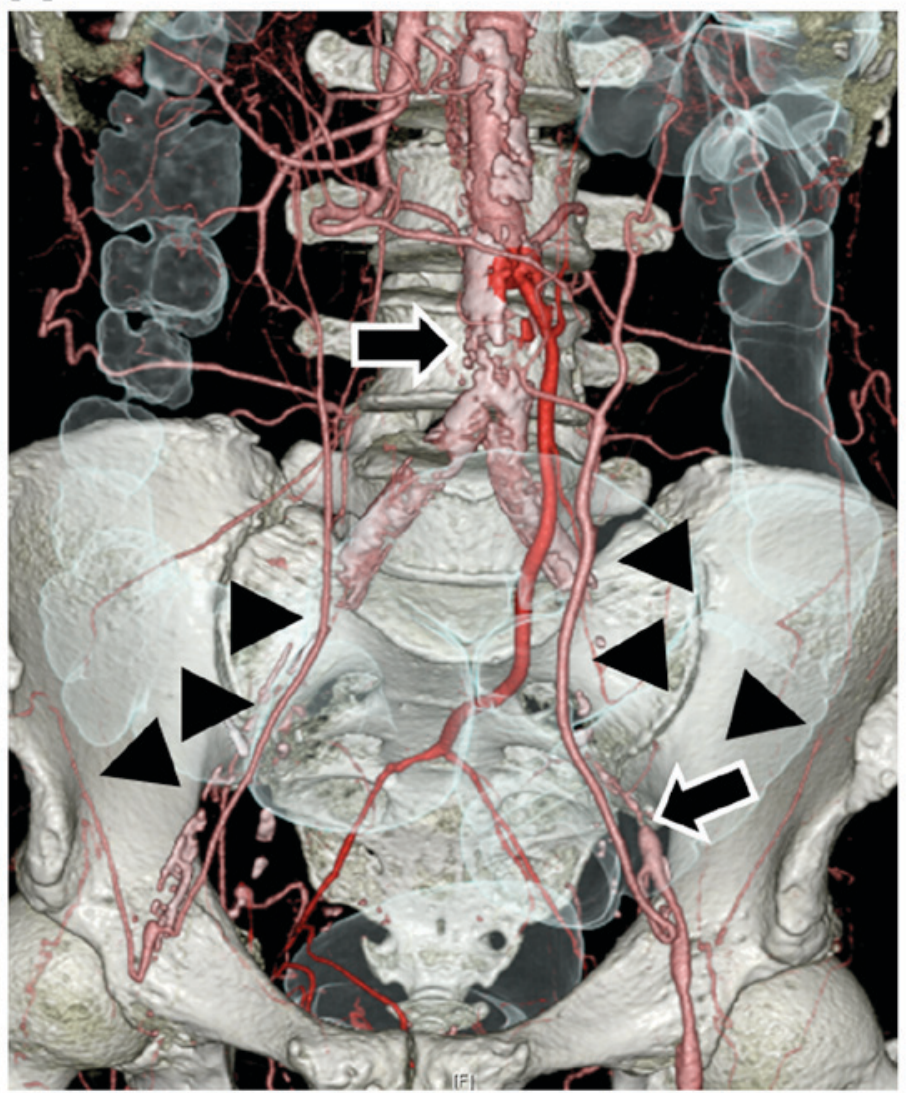

B
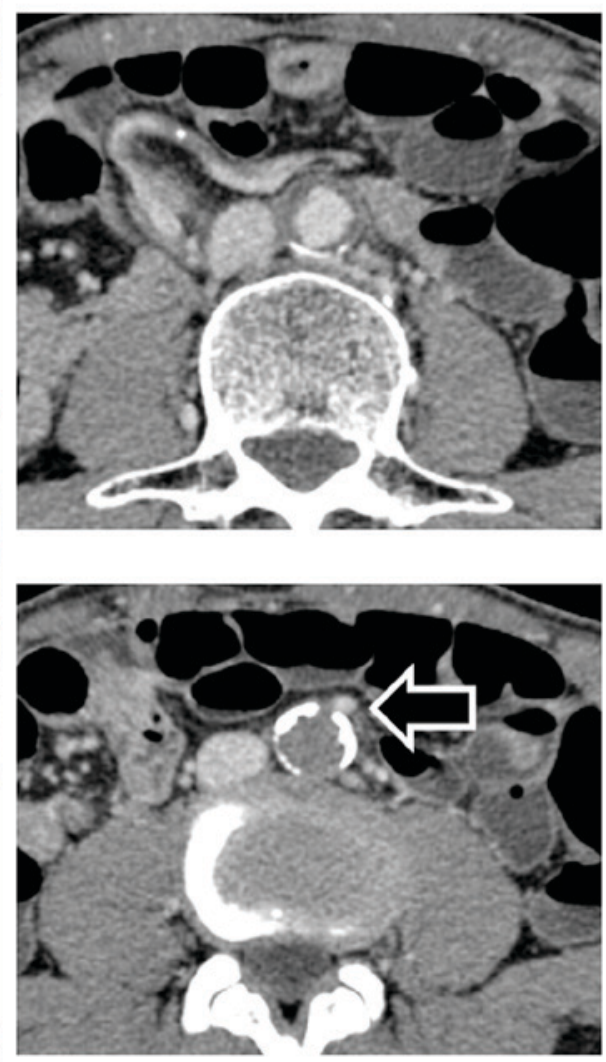

Figure 2. Circulation in case 1. (A) Computed tomography (CT) angiography revealed occlusion of the aorta just below the origin of the inferior mesenteric artery (arrow). The blood flow to the lower limbs was supplied through the inferior epigastric arteries and deep circumflex iliac artery (triangle). (B) In the axial view, the aorta was occluded just below the origin of the inferior mesenteric artery. The arrow indicates the origin of the inferior mesenteric artery.

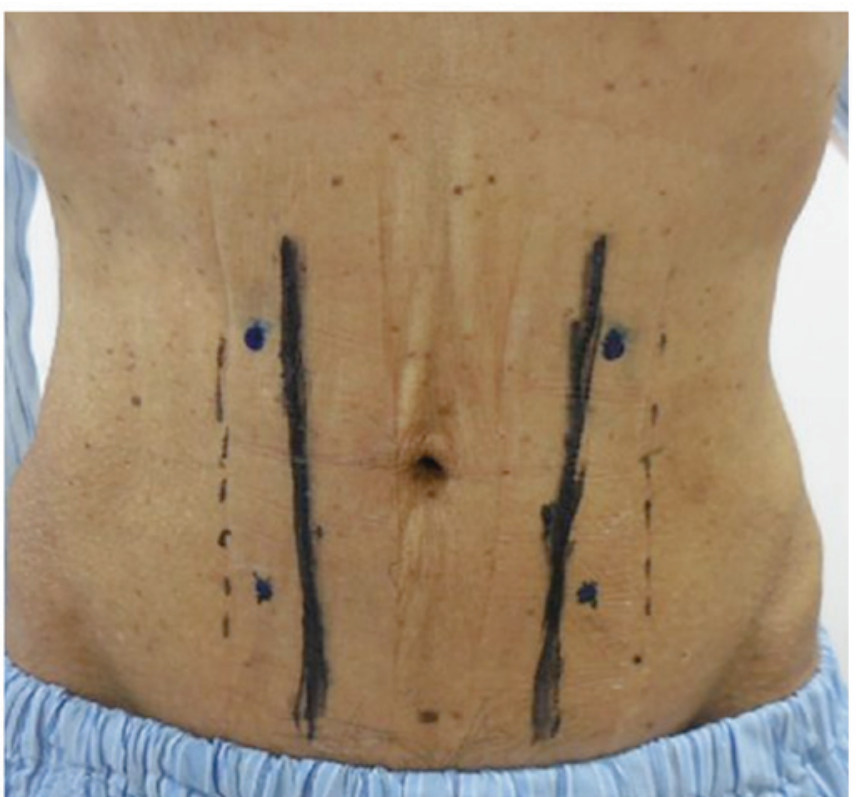

Figure 3. Inferior epigastric arteries and stoma site marking in Case 1. The inferior epigastric arteries were marked with a solid line using ultrasonography.

either intraoperatively or postoperatively. Leriche syndrome is a chronic disease, associated with the development of collateral arteries, and additional artery bypass was unnecessary in all cases. Because there is no report about the surgery of pelvic viscera for the patients with Leriche syndrome, the blood flow of the remnant rectum and the risk of the anastomotic leakage are unknown. The blood flow was supplied from only small arteries in case 1 and the risk of anastomotic leakage seemed to be high. The evaluation of the risk of leakage is an issue in the future.

For patients with Leriche syndrome, the following two pathways are important as collateral arteries to the lower limbs; i) subclavian artery-internal thoracic artery-superior epigastric artery-inferior epigastric artery pathway and ii) subclavian artery-internal thoracic artery-lower intercostals or subcostals arteries-deep circumflex iliac artery pathway (4). CT angiography can aid surgeons in identifying anatomical vascular variations preoperatively $(10,11)$. In all three cases, it was easy to obtain anatomical imaging of these collateral arteries via CT angiography. As trocar insertion has a risk of injuring the inferior epigastric arteries in laparoscopic surgery, the arteries were marked using ultrasonography. While marking was easy because the arteries had enlarged as collateral arteries in the middle and lower abdominal area, there was some difficulty in the upper abdominal area because of some branches. These branches were also confirmed via CT angiography (Figs. 2, 5, and 6). Therefore, the trocar was inserted in the middle or lower abdominal area according to the preoperative marking. The ABI did not worsen in any case, and axillary-femoral artery bypass was unnecessary. Because 
A $\quad$ B
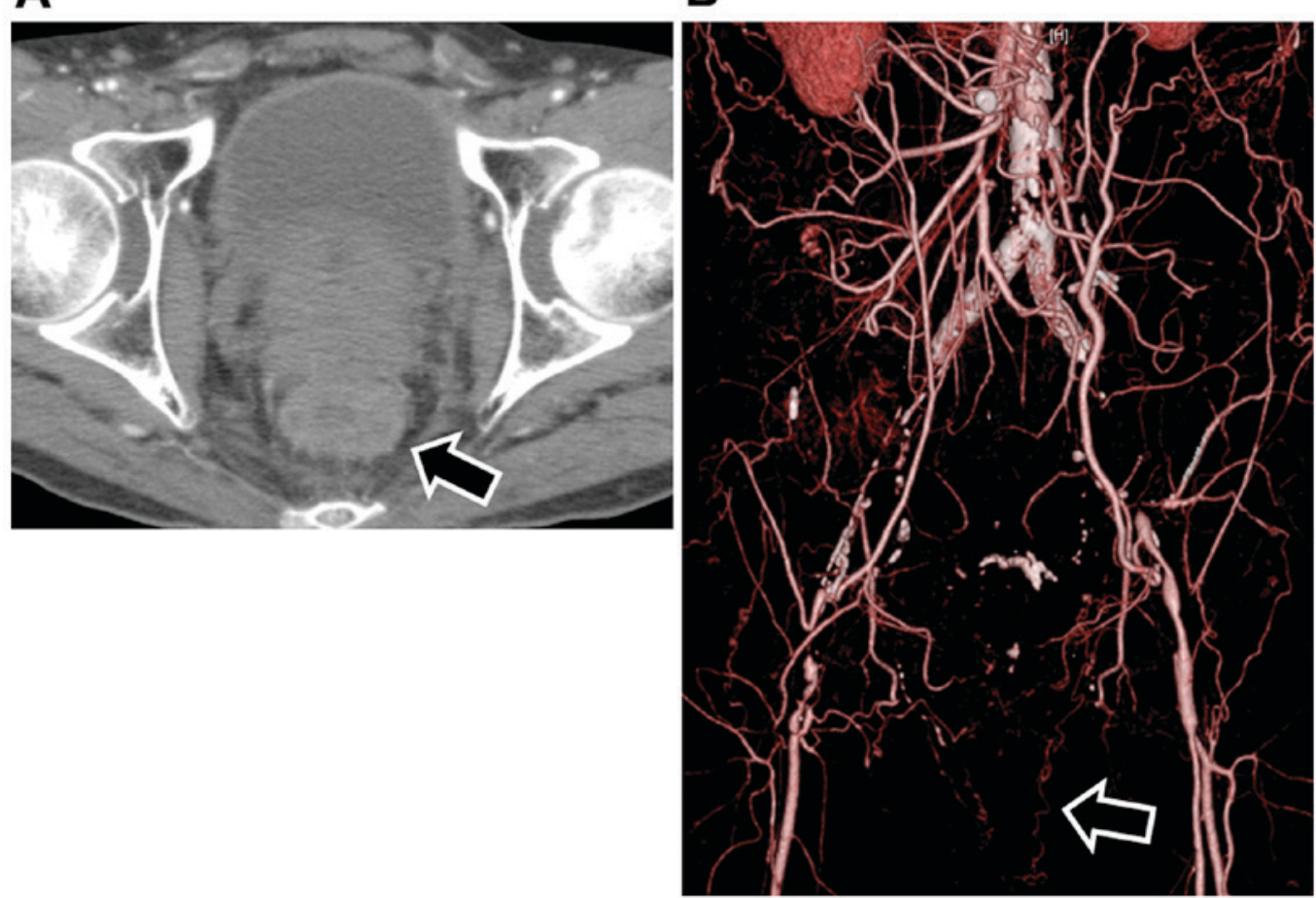

Figure 4. Postoperative circulation in Case 1. (A) The CT scan performed 6 months after the surgery revealed that the enhancement of the remnant rectum remained (arrow). (B) The blood flow was supplied from the small collateral artery (arrow).

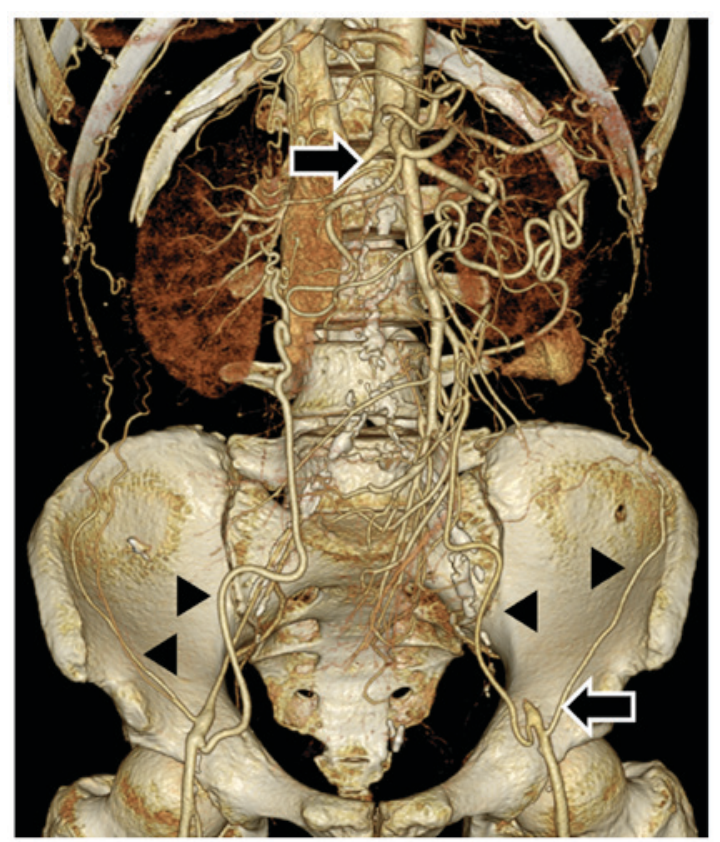

Figure 5. Circulation in case 2. Computed tomography angiography revealed occlusion of the aorta just below the origin of the renal arteries (arrow). The blood flow to the lower limbs was supplied through the inferior epigastric arteries and deep circumflex iliac artery (triangle).

the inferior epigastric arteries were sometimes meandering, such as in case 2 , it is important to obtain images via CT angiography and to mark these arteries using ultrasonography.

The incidence of arteriosclerosis is increasing. It is important to visualize the image the blood flow via CT angiography and to mark collateral arteries using ultrasonography

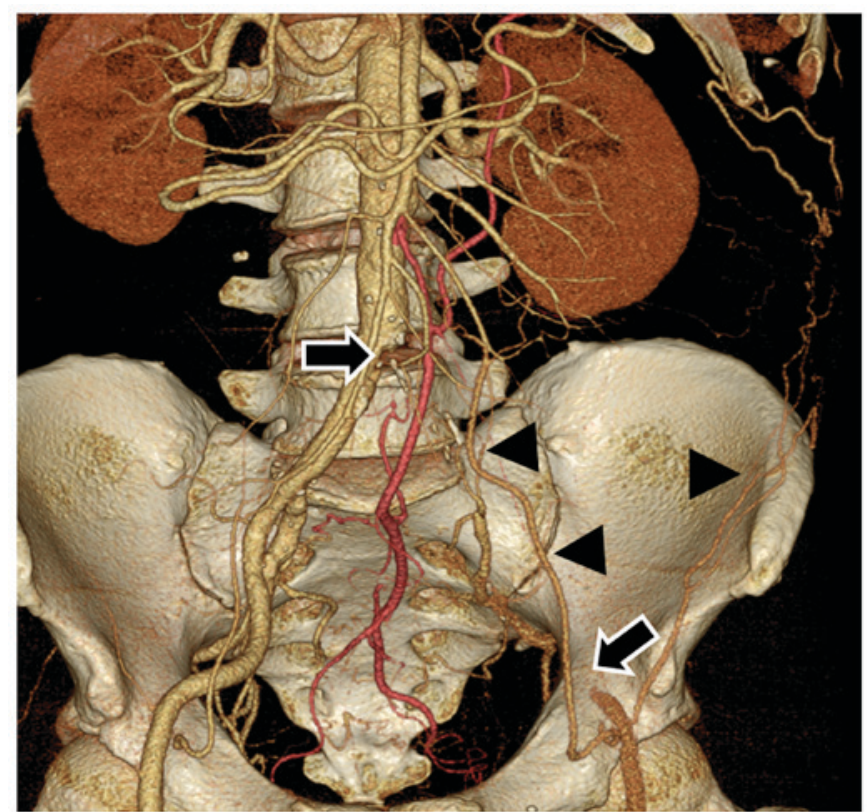

Figure 6. Circulation in case 3. Computed tomography angiography revealed occlusion of the left common iliac artery (arrow). The blood flow to the left lower limb was supplied through the inferior epigastric arteries and deep circumflex iliac artery (triangle).

preoperatively in patients with Leriche syndrome for whom laparoscopic surgery was planned for colorectal cancer.

\section{Acknowledgements}

We would like to thank Editage for English language editing. 


\section{References}

1. Leriche R and Morel A: The syndrome of thrombotic obliteration of the aortic bifurcation. Ann Surg 127: 193-206, 1948.

2. Friedman SA, Holling HE and Roberts B: Etiologic factors in aortoiliac and femoropopliteal vascular disease. The leriche syndrome. N Engl J Med 271: 1382-1385, 1964.

3. Frederick M, Newman J and Kohlwes J: Leriche syndrome. J Gen Intern Med 25: 1102-1104, 2010.

4. Wooten C, Hayat M, du Plessis M, Cesmebasi A, Koesterer M, Daly KP, Matusz P, Tubbs RS and Loukas M: Anatomical significance in aortoiliac occlusive disease. Clin Anat 27: 1264-1274, 2014.

5. Kingham TP and Pachter HL: Colonic anastomotic leak: Risk factors, diagnosis, and treatment. J Am Coll Surg 208: 269-278, 2009.

6. James DR, Ris F, Yeung TM, Kraus R, Buchs NC, Mortensen NJ and Hompes RJ: Fluorescence angiography in laparoscopic low rectal and anorectal anastomoses with pinpoint perfusion imaging-a critical appraisal with specific focus on leak risk reduction. Colorectal Dis 17 (Suppl 3): S16-S21, 2015.
7. Kawada K, Hasegawa S, Wada T, Takahashi R, Hisamori S Hida K and Sakai Y: Evaluation of intestinal perfusion by ICG fluorescence imaging in laparoscopic colorectal surgery with DST anastomosis. Surg Endosc 31: 1061-1069, 2017.

8. Koh FH and Tan KK: Fluorescent angiography used to evaluate the perfusion status of anastomosis in laparoscopic anterior resection. Ann Surg Oncol 23 (Suppl 5): 692, 2016.

9. Järvinen O, Laurikka J, Sisto T and Tarkka MR: Intestinal ischemia following surgery for aorto-iliac disease. A review of 502 consecutive aortic reconstructions. Vasa 25: 148-155, 1996.

10. Murono K, Kawai K, Kazama S, Ishihara S, Yamaguchi H, Sunami E, Kitayama J and Watanabe T: Anatomy of the inferior mesenteric artery evaluated using 3-dimensional CT angiography. Dis Colon Rectum 58: 214-219, 2015.

11. Murono K, Kawai K, Ishihara S, Otani K, Yasuda K, Nishikawa T, Tanaka T, Kiyomatsu T, Hata K, Nozawa H, et al: Evaluation of the vascular anatomy of the right-sided colon using three-dimensional computed tomography angiography: A single-center study of 536 patients and a review of the literature. Int J Colorectal Dis 31: 1633-1638, 2016. 